\title{
Application of Britto-Cachazo-Feng-Witten recursion relations and the Feynman-tree theorem to the four-gluon amplitude with all plus helicities
}

\author{
M. Maniatis* \\ Centro de Ciencias Exactas, Universidad del Bío-Bío, Casilla 447, Chillán, Chile
}

(Received 14 August 2019; published 27 November 2019)

\begin{abstract}
Recently, it has been shown that in gauge theories amplitudes to any perturbation order can be obtained by gluing together simple three-point on-shell amplitudes. These three-point amplitudes, in turn, are fixed by locality and Lorentz invariance. This factorization into three-point on-shell amplitudes follows from the Britto-Cachazo-Feng-Witten recursion relations and the Feynman-tree theorem. In an explicit example, that is, the four-gluon amplitude with all plus helicities, we illustrate the method. In a conventional calculation, this amplitude corresponds to one-loop box diagrams.
\end{abstract}

DOI: 10.1103/PhysRevD.100.096022

\section{INTRODUCTION}

A milestone in the understanding of scattering amplitudes are the Britto-Cachazo-Feng-Witten (BCFW) recursion relations [1,2]: By an analytic continuation of the external momenta, tree amplitudes factorize into elementary building blocks of three-point amplitudes in any gauge theory or in gravity. All external particles as well as inner lines are kept on shell, and gauge invariance is respected by all subdiagrams. An impressive example are the $n$-gluon scattering tree amplitudes, which require in calculations in conventional Feynman diagrams, for instance, for $n=5$ the computation of 25 diagrams; for $n=6$, the number of diagrams increases to 220 . In the maximal helicity violation (MHV) case, that is, with two external gluons $i$ and $j$ carrying minus helicity and all others plus helicity, the amplitude is notably simple:

$A_{n}\left(1^{+}, \ldots, i^{-}, \ldots, j^{-}, \ldots, n^{+}\right)=\frac{\langle i j\rangle}{\langle 12\rangle\langle 23\rangle \ldots\langle n 1\rangle}$

as first conjectured by Parke and Taylor [3]. With the help of the BCFW recursion relations, the amplitude (1.1) for arbitrary $n$ can be easily proven by complete induction; see, for instance, Ref. [4]. However, even though the BCFW recursion relations are very powerful, they are limited to tree diagrams which, of course, form an unphysical subset of diagrams to a certain perturbation order.

\footnotetext{
*maniatis8@gmail.com
}

Published by the American Physical Society under the terms of the Creative Commons Attribution 4.0 International license. Further distribution of this work must maintain attribution to the author(s) and the published article's title, journal citation, and DOI. Funded by SCOAP.
A lot of effort has been spent to extend the recursion relations beyond the tree level, especially to the one-loop order: It has been shown that any amplitude at the one-loop order of a gauge theory can be written as a sum over a finite basis of scalar integrals $I_{m}^{(i)}$ with rational coefficients $c_{m}^{(i)}$ depending only on the outer momenta, with $m=2,3, \ldots, D$, and $D$ denoting the number of dimensions [5-9]:

$A^{1 \text {-loop }}=\sum_{i} c_{D}^{(i)} I_{D}^{(i)}+\sum_{j} c_{D-1}^{(j)} I_{D-1}^{(j)}+\cdots+\sum_{k} c_{2}^{(k)} I_{2}^{(k)}+\mathcal{R}$

and $\mathcal{R}$ denoting a rational term of the kinematic variables. Obviously, the rational term does not have any branch cuts. In the one-loop case, a basis of scalar integrals is known. Applying cuts on both sides of Eq. (1.2), the left-hand side represents products of tree amplitudes and can be calculated. On the right-hand side, the cut contributions can be determined from the known expressions for the scalar integrals. In particular, only those scalar integrals contribute which have the appropriate propagators. In this way, the coefficients $c_{m}^{(i)}$ can be determined, that is, together with the known scalar integrals, the amplitude. The cuts considered are a generalization of the unitary Cutkosky cuts [10], which correspond to Feynman diagrams cut into two separate parts. Generalized cuts denote diagrams where all possible propagator lines are cut, not necessarily splitting the diagram into two parts. Unitary cuts provide the discontinuities of a diagram, but, since the rational part in Eq. (1.2) of the amplitude has no discontinuities in four space-time dimensions, this part is not accessible by unitary cuts. However, in $\mathcal{N}=1$ and $\mathcal{N}=4$ super Yang Mills (SYM) models, it has been shown that also the rational parts can be deduced by unitary cuts $[5,7,11,12]$. This comes from the fact that in these supersymmetric theories 
the rational parts appear always together with logarithms and polylogarithms carrying discontinuities. Another interesting observation is that also in nonsupersymmetric theories the rational part can be determined by treating the momenta not in 4 but, in general, $D$ dimensions [13-15].

The generalized cut methods have been applied, for instance, to the one-loop multigluon amplitudes [16-18]. Let us mention in this context the application of single cuts in $D$ dimensions, calculating the all plus helicity fourand five-gluon amplitudes at one-loop order [19]. New approaches to perform the double cuts have been revealed in Ref. [20] based on Stokes' theorem. In Ref. [21], it has been shown that in SYM theories one-loop amplitudes with arbitrary helicities of the external particles can be derived from the corresponding maximally helicityviolating amplitudes. With respect to single cuts, new techniques have been developed in Ref. [22].

Let us mention the study [23] of the context of the BCFW recursion relations with Veltman's largest time equation [24]. In Ref. [25], it has been shown that the methods of the BCFW recursion relations can be applied to one-loop amplitudes of special helicity configurations.

Feynman has shown decades ago that loops may be opened recursively by the application of generalized cuts [26,27]. Opening the loops means that propagators are replaced by on-shell pairs of particles and antiparticles in the forward limit. The loop integrations are turned into phase-space integrations. The Feynman-tree theorem recursively opens all loops: Any loop is expressed in terms of generalized cut diagrams which reduce the loop order about at least one unit. Iterative application opens all loops and expresses the original Feynman diagram as a sum of tree diagrams.

Many new aspects of the Feynman-tree theorem in multiloop diagrams have been revealed [28-31]. In particular, it has been shown that only a subset of possible generalized cuts contribute in multiloop diagrams.

Recently, it has been shown [32-34] that the application of the Feynman-tree theorem followed by the BCFW recursion allows one to factorize amplitudes at any loop order in terms of elementary three-point amplitudes in gauge theories. In general, an $n$-loop Feynman diagram is turned into a set of tree diagrams after $n$ iteration steps of the Feynman-tree theorem. Eventually, arriving at a form with all loops opened, the tree amplitudes can be factorized by the BCFW recursion relations. In particular, the method is not limited to a certain perturbation order, and gauge invariance is respected by all subamplitudes.

The application of the Feynman-tree theorem followed by the BCFW recursion relations can be reversed, and the amplitudes be constructed by gluing together elementary on-shell three-point amplitudes. Following the BCFW recursion relations, the outer momenta have to be deformed, that is, analytically continued, in order to keep internal lines on shell without violating momentum conservation.
To a certain perturbation order, all possible tree diagrams have to be considered. Following the Feynman-tree theorem, in this process particle-antiparticle pairs in the forward limit have to be taken into account. These pairs are unobservable but contribute, in general, to the corresponding perturbation order. Over the phase space of the unobservable particle pairs in the forward limit has to be integrated. The singularities originating from the particles in the forward limit can be regularized dimensionally.

Since the three-point scattering amplitudes follow, apart from a coupling constant, from locality and little-group scaling, this means that scattering amplitudes eventually result from these first principles along with unitarity. Moreover, every single contributing amplitude is manifestly gauge invariant. Let us note that in a gauge theory like QCD the four-gluon vertex is redundant, since it follows automatically from gluing together three-point on-shell amplitudes.

We shall illustrate the method in an explicit example, the one-loop four-gluon all plus helicity amplitude. We show how we can express this loop amplitude in terms of threepoint on-shell amplitudes glued together. This amplitude provides an excellent framework to study the methods, since it is rather simple but reveals the main steps of the calculation.

\section{GLUING TOGETHER ON-SHELL SUBAMPLITUDES}

We want to consider the all plus helicity four-gluon amplitude $A_{4}\left(1^{+}, 2^{+}, 3^{+}, 4^{+}\right)$. In a conventional Feynman diagram calculation to lowest order, this amplitude follows from a one-loop diagram. For a complex scalar $(s)$ circulating in the loop, this amplitude reads [13,35]

$$
A_{4}^{s, 1-\operatorname{loop}}\left(1^{+}, 2^{+}, 3^{+}, 4^{+}\right)=\frac{i}{(4 \pi)^{2-\epsilon}} \frac{[12][34]}{\langle 12\rangle\langle 34\rangle} I_{4}
$$

with

$$
\begin{aligned}
I_{4}= & -i(4 \pi)^{2-\epsilon} \int \frac{d^{-2 \epsilon} \mu}{(2 \pi)^{-2 \epsilon}} \mu^{4} \\
& \times \int \frac{d^{4} l}{(2 \pi)^{4}} \frac{1}{\left(l_{1}^{2}-\mu^{2}\right)\left(l_{2}^{2}-\mu^{2}\right)\left(l_{3}^{2}-\mu^{2}\right)\left(l_{4}^{2}-\mu^{2}\right)} \\
= & -\frac{1}{6}+\mathcal{O}(\epsilon) .
\end{aligned}
$$

The propagator momenta in the loop are given by

$l_{1}=l, \quad l_{2}=l-p_{1}, \quad l_{3}=l-p_{1}-p_{2}$,

$l_{4}=l-p_{1}-p_{2}-p_{3}=l+p_{4}$.

With respect to the Weyl spinors, we follow the notation of the review in Ref. [4]. We shall now show how to decompose this one-loop four-point Feynman diagram into 
basic building blocks of $A_{3}$ on-shell subamplitudes. The inversion of this procedure is then exactly the process of gluing together elementary $A_{3}$ on-shell amplitudes.

First, let us note that the all plus helicity tree amplitude for $n$ gluons vanishes:

$$
A_{n}\left(1^{+}, 2^{+}, \ldots, n^{+}\right)=0 .
$$

This can be seen, for instance, from the factorization via the BCFW recursion relations eventually into three-point amplitudes of the kind $A_{3}\left(1^{+}, 2^{+}, 3^{+}\right)$which vanish, since all gluons carry plus helicities. Because of momentum conservation, the only nonvanishing three-point on-shell amplitudes are the MHV or anti-MHV amplitudes with complex momenta.

Therefore, nonvanishing four-gluon amplitudes can arise only beyond the tree level. Furthermore, since there is no counterterm available, which would be proportional to the tree amplitude, there cannot appear divergences at the oneloop order. The logarithms (respectively, polylogarithms) appear with the singularities. Therefore, we expect the oneloop amplitude to be a rational expression in the kinematic variables.

Applying supersymmetric Ward identities, it has been shown [14] that, in $\mathcal{N}=4$ and $\mathcal{N}=1 \mathrm{SYM}$, the relation (2.4) holds not only at the tree level, but to all orders in perturbation theory. For instance, in $\mathcal{N}=4 \mathrm{SYM}$, the oneloop gluon amplitude consists in one contribution with a gluon ( $g$, spin 1) in the loop, besides four Weyl fermions $(f$, spin $1 / 2)$, as well as three complex scalars $(s$, spin 0$)$ :

$$
A_{n}^{\mathcal{N}=4,1 \text {-loop }}=A_{n}^{g, 1-\text { loop }}+4 A_{n}^{f, 1 \text {-loop }}+3 A_{n}^{s, 1-\text { loop }} .
$$

In contrast, in $\mathcal{N}=1 \mathrm{SYM}$, we have in the loop to consider one gluon paired with one Weyl fermion:

$$
A_{n}^{\mathcal{N}=1,1 \text {-loop }}=A_{n}^{g, 1-\text { loop }}+A_{n}^{f, 1 \text {-loop }} .
$$

Since the four-gluon amplitudes vanish in $\mathcal{N}=4$ and $\mathcal{N}=1$ SYM, that is, $A_{4}^{\mathcal{N}=4,1 \text {-loop }}\left(1^{+}, 2^{+}, 3^{+}, 4^{+}\right)=0$ and $A_{4}^{\mathcal{N}=1,1 \text {-loop }}\left(1^{+}, 2^{+}, 3^{+}, 4^{+}\right)=0$, we arrive at the relations [14]

$$
\begin{aligned}
A_{4}^{g, 1-\text { loop }}\left(1^{+}, 2^{+}, 3^{+}, 4^{+}\right) & =-A_{4}^{f, 1-\text { loop }}\left(1^{+}, 2^{+}, 3^{+}, 4^{+}\right) \\
& =A_{4}^{s, 1-\text { loop }}\left(1^{+}, 2^{+}, 3^{+}, 4^{+}\right) .
\end{aligned}
$$

Therefore, it suffices to focus on the calculation of the amplitude corresponding to a complex scalar $(s)$ in the loop. Considering this loop diagram, we will encounter as elementary building blocks the gluon-scalar-scalar on-shell three-point amplitude. This amplitude is, apart from a coupling constant, fixed by little group scaling and from locality. For simplicity, we set the coupling constant to one. Depending on the helicity of the gluon, these elementary three-point amplitudes read (see, for instance, Refs. [36,37])
$A_{3}\left(1,2^{+}, 3\right)=\frac{\langle q|1| 2]}{\langle q 2\rangle}, \quad A_{3}\left(1,2^{-}, 3\right)=-\frac{\langle 2|1| q]}{[q 2]}$

with complex momenta 1 and 3 for the scalars and the gluon with momentum 2 and $q$ an arbitrary linearly independent null vector. As we will see later with respect to the BCFW recursion relations, it is convenient to choose this vector $q$ to be the shifted momentum of the opposite side of the factorized amplitude.

We shall now show how the conventional Feynman diagram can be factorized into the elementary three-point amplitudes (2.8). We start considering the one-loop box integral as a conventional Feynman diagram, and in a first step we apply the Feynman-tree theorem [26,27]. The Feynman-tree theorem, valid at any loop order and for arbitrary dimensions $D$, reads, considering a loop of a Feynman diagram with loop momentum $l$,

$$
\begin{aligned}
0= & \int \frac{d^{D} l}{(2 \pi)^{D}} N(l) \prod_{i}\left\{G_{F}^{(i)}\left(l-p_{1}-\cdots-p_{i-1}\right)\right. \\
& \left.-2 \pi \delta^{(+)}\left(\left(l-p_{1}-\cdots-p_{i-1}\right)^{2}-\mu_{i}^{2}\right)\right\}
\end{aligned}
$$

where $G_{F}^{(i)}(p)=\frac{i}{p^{2}-\mu_{i}^{2}+i \epsilon}$ is the $i$ th propagator in the loop and $N(l)$ is the numerator depending on the details of the model. From the expansion of the product on the right-hand side of Eq. (2.9), we see that we can express the original amplitude in terms of all different generalized cut diagrams. The generalized cuts are given by the replacements of the propagators by $-2 \pi$ times the $\delta^{(+)}$ distributions:

$$
\frac{i}{p^{2}-\mu^{2}+i \epsilon} \rightarrow-2 \pi \delta^{(+)}\left(p^{2}-\mu^{2}\right) .
$$

The cut, that is, the $\delta^{(+)}$distribution, puts the momentum originating from the propagator on shell. Iteratively, we can open all the loops and archive a set of cut diagrams which are all tree diagrams. Applying the Feynman-tree theorem (2.9) to the one-loop four-gluon amplitude (2.1), we get by one iteration step four single-cut diagrams, six double-cut diagrams, four triple-cut diagrams, and one quadruple-cut diagram. However, since the outer particles are on shell, the quadruple cut and all triple cuts vanish immediately, since they isolate at least one three-point amplitude with real momenta. From the six double-cut diagrams, only two survive as well as all four single-cut diagrams. The double-cut diagrams which do not leave any isolated on shell $A_{3}$ amplitude are the horizontal and the vertical cuts of the diagram. All not immediately vanishing single and double cuts which arise from the Feynman-tree theorem are shown in Fig. 1.

Since we are applying the Feynman tree theorem to a one-loop Feynman diagram, there is only one recursion step needed in order to get tree diagrams. In general, an 


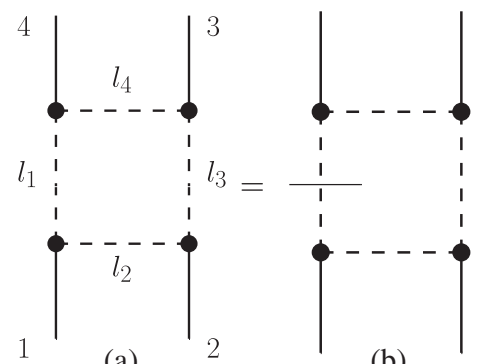

(a) (b)

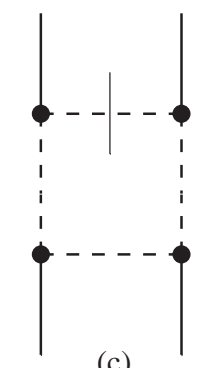

(c)

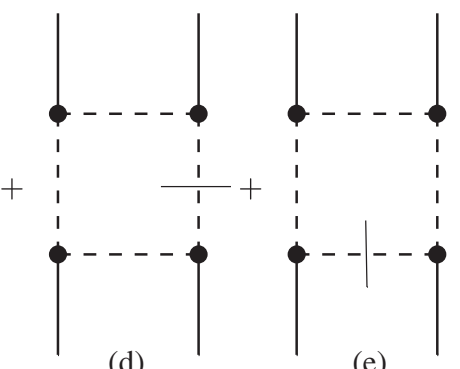

(d)

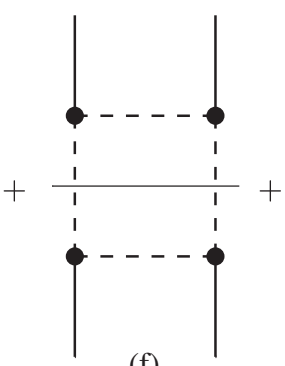

(f)

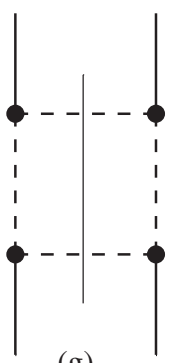

(g)

FIG. 1. The Feynman box diagram and its decomposition in terms of generalized cut diagrams. All other cut diagrams vanish, because they isolate a vanishing on-shell subamplitude $A_{3}$ with real momenta.

$n$-loop Feynman diagram requires $n$ recursion steps in order to open all loops. The number of cut diagrams for a loop with $i$ propagators is $2^{i}-1$. In general, only a subset of these diagrams contributes; respectively, there are simple relations among these diagrams, as we will explicitly see in the amplitude considered here. Note that the double cuts appearing on the right-hand side in Fig. 1 correspond to the usual unitarity cuts, where the whole diagram is split into two parts.

We see that all four single-cut diagrams are related by changing cyclically $1 \rightarrow 2 \rightarrow 3 \rightarrow 4 \rightarrow 1$. Similarly, the two double-cut diagrams, that is, unitary cut diagrams, are related by exchanging $2 \leftrightarrow 4$. This, in terms of the Mandelstam invariants $s=\left(p_{1}+p_{2}\right)^{2}$ and $t=\left(p_{2}+p_{3}\right)^{2}$, corresponds to $s \leftrightarrow t$.

We work in the four-dimensional helicity scheme [35]; that is, the external momenta are kept four dimensional, while the loop momenta are treated in $D$ dimensions. As has been shown, the $D$-dimensional momentum can be split into its four $l$ and $(-2 \epsilon) \mu$ components, that is, $l \rightarrow l+\mu$. The integration measure can, therefore, be replaced by $d^{D} l \rightarrow$ $d^{-2 \epsilon} \mu d^{4} l$ and the propagators in the amplitudes transform as $(l-p)^{2} \rightarrow(l-p)^{2}-\mu^{2}$. We get a four-dimensional loop integration with an artificial mass parameter $\mu$.

Let us start considering the two double-cut diagrams. As we have argued above, from the six double-cut diagrams we have four which vanish immediately, since they isolate an on-shell three-point amplitude $A_{3}$, vanishing for real momenta. The remaining two are shown in Fig. 1 on the right-hand side with the indicated unitary cuts, that is, in our case, double cuts. As mentioned before, the two double-cut diagrams are related by exchanges of outer momenta. We therefore calculate only one of them, that is, the diagram with the momenta $l_{1}$ and $l_{3}$ cut as shown in the next-to-last diagram in Fig. 1, and get

$$
\begin{aligned}
& A_{4, \text { cut13 }}^{\text {1-loop }}\left(1^{+}, 2^{+}, 3^{+}, 4^{+}\right) \\
& =\int \frac{d^{-2 \epsilon} \mu}{(2 \pi)^{-2 \epsilon}} \int \frac{d^{4} l}{(2 \pi)^{4}}(-2 \pi) \delta^{(+)}\left(l_{1}^{2}-\mu^{2}\right)(-2 \pi) \\
& \quad \times \delta^{(+)}\left(l_{3}^{2}-\mu^{2}\right) A_{4}\left(-l_{1}, 1^{+}, 2^{+}, l_{3}\right) A_{4}\left(-l_{3}, 3^{+}, 4^{+}, l_{1}\right) .
\end{aligned}
$$

Since we want to decompose the amplitude into its elementary $A_{3}$ building blocks, it remains to factorize the four-point amplitudes which appear in the integrand. In fact, these amplitudes are tree amplitudes, and, therefore, we can apply the BCFW recursion relations. We consider a general four-point amplitude of two gluons and two scalars, where the two gluons carry plus helicities, that is, $A_{4}\left(1,2^{+}, 3^{+}, 4\right)$. We apply a $[2,3\rangle$ shift yielding with the propagator momentum $P=p_{1}+p_{2}$ :

$$
\begin{aligned}
A_{4}\left(1,2^{+}, 3^{+}, 4\right) & =A_{3}\left(\hat{2}^{+}, 1,-\hat{P}\right) \frac{1}{P^{2}-\mu^{2}} A_{3}\left(\hat{P}, \hat{3}^{+}, 4\right) \\
& =-\frac{\langle\hat{3}|\hat{P}| \hat{2}]}{\langle\hat{3} \hat{2}\rangle} \frac{1}{P^{2}-\mu^{2}} \frac{\langle\hat{2}|4| \hat{3}]}{\langle\hat{2} \hat{3}\rangle} \\
& =\frac{\mu^{2}[23]}{\langle 23\rangle\left(P^{2}-\mu^{2}\right)}
\end{aligned}
$$

where we used cyclicity of the amplitudes and have plugged in the elementary three-point amplitude (2.8) with convenient reference vectors.

We insert the factorization (2.12) into Eq. (2.11) and get

$$
\begin{aligned}
& A_{4, \text { cut13 }}^{1 \text {-loop }}\left(1^{+}, 2^{+}, 3^{+}, 4^{+}\right) \\
& =\frac{[12][34]}{\langle 12\rangle\langle 34\rangle} \int \frac{d^{-2 \epsilon} \mu}{(2 \pi)^{-2 \epsilon}} \mu^{4} \\
& \quad \times \int \frac{d^{4} l}{(2 \pi)^{4}} \frac{(-2 \pi) \delta^{(+)}\left(l_{1}^{2}-\mu^{2}\right)(-2 \pi) \delta^{(+)}\left(l_{3}^{2}-\mu^{2}\right)}{\left(l_{2}^{2}-\mu^{2}\right)\left(l_{4}^{2}-\mu^{2}\right)} .
\end{aligned}
$$

Writing the loop integral in terms of a double integral with a four-dimensional delta distribution,

$$
\begin{aligned}
\int \frac{d^{4} l}{(2 \pi)^{4}}= & \int \frac{d^{4} l_{1}}{(2 \pi)^{4}} \frac{d^{4} l_{3}}{(2 \pi)^{4}}(2 \pi)^{4} \\
& \times \delta^{(4)}\left(p_{1}+p_{2}-l_{1}-l_{3}\right)
\end{aligned}
$$

and performing the integrations over the energy components of $l_{1}$ and $l_{3}$ with the help of the $\delta^{(+)}$distributions, this amplitude corresponds to a phase-space integral over two 
unobservable pairs of particles. On the other hand, from the Cutkosky cutting rules, this phase-space integral equals twice the imaginary part of the corresponding box integral. However, we know that the imaginary parts can originate only from discontinuities of logarithms and polylogarithms. Since the scalar box integral has to be a rational function, we conclude that this integral vanishes.

The $t$ channel double cut follows from exchanging momenta $2 \leftrightarrow 4$. Considering the kinematic factor, we find from momentum conservation $[12]=\langle 41\rangle[12] /\langle 41\rangle=$ $-\langle 4|1| 2] /\langle 41\rangle=\langle 4|3| 2] /\langle 41\rangle=-\langle 43\rangle[32] /\langle 41\rangle$, and similarly we have $[34]=-\langle 21\rangle[14] /\langle 23\rangle$; that is, we have cyclic invariance of the kinematic factor:

$$
\frac{[12][34]}{\langle 12\rangle\langle 34\rangle}=\frac{[23][41]}{\langle 23\rangle\langle 41\rangle} .
$$

From this symmetry, we find that the two double-cut integrals are equal; that is, both vanish.

We now want to compute the single-cut diagram with the cut applied to the propagator $l_{1}$ between the gluons 1 and 4 as shown in the first term on the right-hand side of the equation in Fig. 1. All other single-cut diagrams follow from cyclic permutations of the outer momenta. The diagram with a single cut of the $l_{1}$ propagator reads

$$
\begin{aligned}
& A_{4, \text { cut } 1}^{1 \text {-loop }}\left(1^{+}, 2^{+}, 3^{+}, 4^{+}\right) \\
& =\int \frac{d^{-2 \epsilon} \mu}{(2 \pi)^{-2 \epsilon}} \int \frac{d^{4} l}{(2 \pi)^{4}}(-2 \pi) \delta^{(+)}\left(l_{1}^{2}-\mu^{2}\right) \\
& \quad \times A_{6}\left(-l_{1}, 1^{+}, 2^{+}, 3^{+}, 4^{+}, l_{1}\right) .
\end{aligned}
$$

The tree amplitude in the integrand is a six-point amplitude with one unobservable particle-antiparticle pair of complex scalars besides four-gluons. The two complex scalars are in the forward limit. We proceed decomposing this six-point amplitude applying the BCFW recursion relations. Since the propagators appear with the mass parameter $\mu$, we have to apply the recursion relations adopted to the massive case [36]. We follow the calculation performed in Ref. [36], where we here have the case of the two complex scalars in the forward limit. Applying the BCFW recursion relations, we have to sum over all possible factorizations, corresponding to each propagator in turn on shell. We choose a $[1,2\rangle$ shift with only one nonvanishing contribution as shown in Fig. 2. All other single cuts leave both shifted momenta on one side of the BCFW factorization and, therefore, vanish. We get

$$
\begin{aligned}
& A_{6}\left(-l_{1}, 1^{+}, 2^{+}, 3^{+}, 4^{+}, l_{1}\right) \\
& \quad=A_{3}\left(-l_{1}, \hat{1}^{+}, \hat{l}_{2}\right) \frac{1}{l_{2}^{2}-\mu^{2}} A_{5}\left(-\hat{l}_{2}, \hat{2}^{+}, 3^{+}, 4^{+}, l_{1}\right) .
\end{aligned}
$$

The $[1,2\rangle$ shift reads explicitly

$$
\begin{aligned}
\mid \hat{1}]=\mid 1]+z \mid 2], & \mid \hat{2}]=\mid 2], \\
|\hat{2}\rangle=|2\rangle-z|1\rangle, & |\hat{1}\rangle=|1\rangle,
\end{aligned}
$$

where the complex number $z$ is given by

$$
z=\frac{l_{2}^{2}-\mu^{2}}{\left\langle 2\left|l_{2}\right| 1\right]}
$$

One of the amplitudes coming from the BCFW recursion (2.17) is already an elementary three-point amplitude $A_{3}$. In a second recursion step, we factorize the five-point amplitude $A_{5}\left(1,2^{+}, 3^{+}, 4^{+}, 5\right)$, where we choose conveniently again a shift of the two neighboring gluons, that is, a $|2,3\rangle$ shift:

$$
\begin{aligned}
& A_{5}\left(1,2^{+}, 3^{+}, 4^{+}, 5\right) \\
& \quad=A_{3}\left(1, \hat{2}^{+}, \hat{P}\right) \frac{1}{P^{2}-\mu^{2}} A_{4}\left(-\hat{P}, \hat{3}^{+}, 4^{+}, 5\right) .
\end{aligned}
$$

With another BCFW iteration step to factorize the fourpoint amplitude with two complex scalars (2.12) into threepoint amplitudes, we get

$$
\begin{aligned}
A_{5}\left(1,2^{+}, 3^{+}, 4^{+}, 5\right)= & \mu^{2} \frac{\langle\hat{3}|1| \hat{2}]}{\langle\hat{3} \hat{2}\rangle} \frac{1}{P^{2}-\mu^{2}} \frac{1}{\left(p_{4}+p_{5}\right)^{2}-\mu^{2}} \frac{[\hat{3} 4]}{\langle\hat{3} 4\rangle} \\
= & \frac{\mu^{2}}{\left[\left(p_{1}+p_{2}\right)^{2}-\mu^{2}\right]\left[\left(p_{5}+p_{4}\right)^{2}-\mu^{2}\right]} \\
& \times \frac{[42]\langle 21\rangle[12]+[43]\langle 31\rangle[12]}{\langle 23\rangle\langle 34\rangle}
\end{aligned}
$$

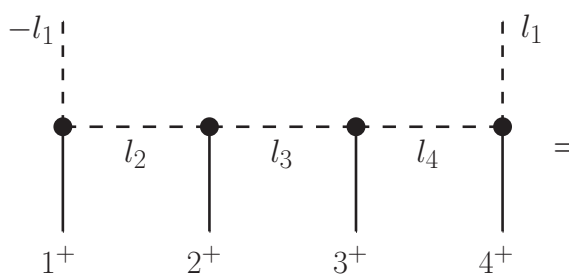

(a)

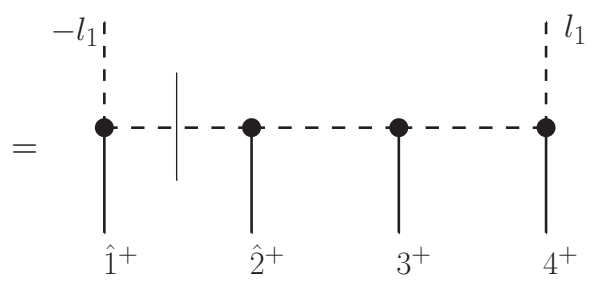

(b)

FIG. 2. The single cut of the box diagram and its factorization. For a $[1,2\rangle$ shift, all other cut diagrams vanish, because they leave both shifted momenta on one side of the propagator. 
and, in turn (see also Ref. [36]),

$$
\begin{aligned}
A_{6} & \left(-l_{1}, 1^{+}, 2^{+}, 3^{+}, 4^{+}, l_{1}\right) \\
& =\frac{\mu^{4}}{\left(l_{1}^{2}-\mu^{2}\right)\left(l_{2}^{2}-\mu^{2}\right)\left(l_{3}^{2}-\mu^{2}\right)} \frac{[12][34]}{\langle 12\rangle\langle 34\rangle} .
\end{aligned}
$$

We now plug this result into Eq. (2.16) and eventually get

$$
\begin{aligned}
& A_{4, \text { cut } 1}^{1 \text {-loop }}\left(1^{+}, 2^{+}, 3^{+}, 4^{+}\right) \\
& =\frac{[12][34]}{\langle 12\rangle\langle 34\rangle} \int \frac{d^{-2 \epsilon} \mu}{(2 \pi)^{-2 \epsilon}} \mu^{4} \\
& \quad \times \int \frac{d^{4} l}{(2 \pi)^{4}} \frac{(-2 \pi) \delta^{(+)}\left(l_{1}^{2}-\mu^{2}\right)}{\left(l_{2}^{2}-\mu^{2}\right)\left(l_{3}^{2}-\mu^{2}\right)\left(l_{4}^{2}-\mu^{2}\right)} .
\end{aligned}
$$

It remains to calculate the integral, where we conveniently go back to $D$ dimensions:

$$
I=\int \frac{d^{D} l}{(2 \pi)^{D}} \frac{-2 \pi \delta^{(+)}\left(l_{1}^{2}\right)}{\left(l_{2}^{2}+i \epsilon\right)\left(l_{3}^{2}+i \epsilon\right)\left(l_{4}^{2}+i \epsilon\right)} .
$$

We compute this integral with the help of Schwinger parameters (see also Ref. [31] for a similar computation):

$\frac{i}{x+i \epsilon} \rightarrow \int_{0}^{\infty} d a e^{i a x}, \quad 2 \pi \delta(x) \rightarrow \int_{-\infty}^{\infty} d a e^{i a x}$,

and get, by integrating over the common Schwinger parameter, over the parameter corresponding to the delta distribution, and over the momentum $l$ in $D$ dimensions,

$$
\begin{aligned}
I= & \frac{-i \pi^{D / 2}}{2(2 \pi)^{D}} \Gamma\left(2-\frac{D}{2}\right) \int_{0}^{\infty} d \alpha_{2} d \alpha_{3} d \alpha_{4} \\
& \times\left[s \alpha_{3}\left(\alpha_{2}+\alpha_{3}+\alpha_{4}-1\right)-t \alpha_{2} \alpha_{4}-i \epsilon\right]^{(D / 2)-2}
\end{aligned}
$$

with $s=2 p_{1} p_{2}$ and $t=2 p_{2} p_{3}$. With the substitution $\alpha_{3} \rightarrow \alpha_{3} \alpha_{4}$, integration over $\alpha_{2}$, followed by $\alpha_{4}$, expansion about $D=4$ dimensions, and integrating eventually over $\alpha_{3}$, we get

$I=\frac{-i}{32 \pi^{2}} \frac{1}{6}\left(\frac{s}{s+t}+\frac{s t}{(s+t)^{2}} \log \left(\frac{s}{-t}\right)\right)+\mathcal{O}(D-4)$.

The other single-cut diagrams follow from cyclic permutations $1 \rightarrow 2 \rightarrow 3 \rightarrow 4 \rightarrow 1$, and we see that we get two equal contributions (2.27) and two contributions exchanging $s \leftrightarrow t$. Hence, the kinematic factor exactly cancels in the sum, and the final result we find for the amplitude is

$$
A_{4}^{s}\left(1^{+}, 2^{+}, 3^{+}, 4^{+}\right)=\frac{-i}{16 \pi^{2}} \frac{1}{6} \frac{[12][34]}{\langle 12\rangle\langle 34\rangle}+\mathcal{O}(\epsilon) .
$$

Applying the Feynman-tree theorem followed by the $\mathrm{BCFW}$ recursion relations, we see that we can reproduce the known result (2.1).

\section{DISCUSSION}

We have seen explicitly that the one-loop box diagram in a conventional Feynman diagram calculation can be represented in terms of generalized cut diagrams. All triple and quadruple cuts vanish immediately, since they isolate at least one on-shell three-point amplitude with real momenta. Subsequently applying the BCFW recursion relations, the tree amplitudes factorize into elementary building blocks of three-point amplitudes $A_{3}$.

Reversing the order of the calculation, we can construct the amplitude by gluing together three-point amplitudes. The momenta can kept on shell by their analytic continuation; that is, following the $\mathrm{BCFW}$ recursion relations, whenever we glue together two on-shell subamplitudes, we have to deform the outer momenta accordingly. Conveniently, this can be done by the application of a two-particle shift of two of the external momenta - one out of each subamplitude. To a certain perturbation order, we have to collect all possible constructions of glued on-shell amplitudes. As we have seen in the decomposition, in many cases we encounter vanishing contributions, for instance, when we isolate three-point amplitudes with all gluons carrying minus or plus helicities. To a certain perturbation order, we also have to consider amplitudes with pairs of outer particles in the forward limit, that is, particleantiparticle pairs with opposite momenta and opposite quantum numbers. These pairs are unobservable, since they represent vacuum states. Therefore, these contributions appear in a quite natural way. In the conventional Feynman-diagram approach, these amplitudes correspond to loop diagrams. Let us emphasize that the Feynman-tree theorem is not limited to the one-loop order but holds to any loop order. Therefore, we can apply the method of gluing together elementary building blocks to any perturbation order.

Let us note that, in general, the pairs of particles in the forward limit give rise to singularities. However, performing the calculation of the amplitudes in general $D$ dimensions, we can regularize these singularities.

Some remarks to the gluing process are in order: To a certain perturbation order, we consider all contributions taking unobservable pairs of particles in the forward limit into account. In principle, we may encounter contributions, where we glue together subamplitudes which result in a loop. However, this kind of loop is quite different from a loop in the conventional Feynman-diagram approach. In the amplitude approach, all outer and inner lines are on shell in contrast to Feynman diagrams. As we can see, gluing together tree diagrams to an on-shell loop diagram, we cannot satisfy momentum conservation and on-shellness simultaneously, and, therefore, these contributions vanish 
naturally. We conclude that by gluing together elementary amplitudes we can disregard any loops.

\section{CONCLUSION}

The BCFW recursion relations factorize the tree amplitude of gauge theories into elementary three-point on-shell amplitudes which form the elementary building blocks. These elementary building blocks, in turn, are, apart from a coupling constant, fixed by little group scaling and locality. Since we are considering color-ordered amplitudes, the complete amplitudes, therefore, will depend also on the gauge symmetry of the model.

If we first open the loops by iterative application of the Feynman-tree theorem and then recursively apply the BCFW recursion relations, we can factorize any amplitude to any perturbation order into elementary three-point amplitudes. Reversing the recursion relations, we can construct amplitudes by gluing together on-shell threepoint amplitudes. To a certain perturbation order, we have to consider amplitudes with additional pairs of particles and antiparticles with opposite momenta and opposite quantum numbers, that is, particle pairs in the forward limit corresponding to unobservable vacuum states.

We have applied this method to the four-gluon amplitude with all gluons carrying plus helicities to leading order.
This amplitude corresponds in a conventional calculation to one-loop Feynman diagrams with scalars, fermions, and gluons in the loop. By a hidden supersymmetry, all loop contributions can be related to the contribution with a complex scalar running in the loop. Gluing together threepoint amplitudes $A_{3}$ to the fourth order in the coupling, we have to consider up to four additional particle-antiparticle pairs. However, all contributions with three or four particleantiparticle pairs vanish, because they isolate at least one on-shell three-point amplitude with real momenta. We have seen that, in the case of all plus amplitudes, also the diagrams with two pairs in the forward limit vanish. Eventually, we have computed the contributions with one particle pair in the forward limit. In an explicit calculation of these contributions, we have reproduced the known result for the amplitude. Eventually, let us emphasize that this calculation of a color-ordered amplitude is based only on locality, little group scaling (that is, Lorentz invariance), and unitarity.

\section{ACKNOWLEDGMENTS}

I thank D. Diáz and P. Mastrolia for helpful discussions. The project was supported in part by the UBB project "Materia Obscura y los bosones de Higgs" with No. DIUBB 193209 1/R.
[1] R. Britto, F. Cachazo, and B. Feng, New recursion relations for tree amplitudes of gluons, Nucl. Phys. B715, 499 (2005).

[2] R. Britto, F. Cachazo, B. Feng, and E. Witten, Direct Proof of Tree-Level Recursion Relation in Yang-Mills Theory, Phys. Rev. Lett. 94, 181602 (2005).

[3] S. J. Parke and T. R. Taylor, Amplitude for $n$ Gluon Scattering, Phys. Rev. Lett. 56, 2459 (1986).

[4] H. Elvang and Y.t. Huang, Scattering amplitudes, arXiv: 1308.1697.

[5] Z. Bern, L. J. Dixon, D. C. Dunbar, and D. A. Kosower, One loop n-point gauge theory amplitudes, unitarity and collinear limits, Nucl. Phys. B425, 217 (1994).

[6] W. L. van Neerven and J. A. M. Vermaseren, Large loop integrals, Phys. Lett. 137B, 241 (1984).

[7] S. J. Bidder, N. E. J. Bjerrum-Bohr, D. C. Dunbar, and W. B. Perkins, One-loop gluon scattering amplitudes in theories with $N<4$ supersymmetries, Phys. Lett. B 612, 75 (2005).

[8] C. Anastasiou, R. Britto, B. Feng, Z. Kunszt, and P. Mastrolia, D-dimensional unitarity cut method, Phys. Lett. B 645, 213 (2007).

[9] W. T. Giele, Z. Kunszt, and K. Melnikov, Full one-loop amplitudes from tree amplitudes, J. High Energy Phys. 04 (2008) 049.

[10] R. E. Cutkosky, Singularities and discontinuities of Feynman amplitudes, J. Math. Phys. (N.Y.) 1, 429 (1960).
[11] R. Britto, F. Cachazo, and B. Feng, Generalized unitarity and one-loop amplitudes in $N=4$ super-Yang-Mills, Nucl. Phys. B725, 275 (2005).

[12] Z. Bern, L. J. Dixon, D. C. Dunbar, and D. A. Kosower, Fusing gauge theory tree amplitudes into loop amplitudes, Nucl. Phys. B435, 59 (1995).

[13] Z. Bern and A. G. Morgan, Massive loop amplitudes from unitarity, Nucl. Phys. B467, 479 (1996).

[14] Z. Bern, L. J. Dixon, D. C. Dunbar, and D. A. Kosower, One loop selfdual and $N=4$ superYang-Mills, Phys. Lett. B 394, 105 (1997).

[15] A. Brandhuber, S. McNamara, B. J. Spence, and G. Travaglini, Loop amplitudes in pure Yang-Mills from generalised unitarity, J. High Energy Phys. 10 (2005) 011.

[16] Z. Bern, L. J. Dixon, and D. A. Kosower, On-shell recurrence relations for one-loop QCD amplitudes, Phys. Rev. D 71, 105013 (2005).

[17] Z. Bern, G. Chalmers, L. J. Dixon, and D. A. Kosower, One-Loop n-Gluon Amplitudes with Maximal Helicity Violation via Collinear Limits, Phys. Rev. Lett. 72, 2134 (1994).

[18] G. Mahlon, Multigluon helicity amplitudes involving a quark loop, Phys. Rev. D 49, 4438 (1994).

[19] E. W. Nigel Glover and C. Williams, One-loop gluonic amplitudes from single unitarity cuts, J. High Energy Phys. 12 (2008) 067. 
[20] P. Mastrolia, Double-cut of scattering amplitudes and Stokes' theorem, Phys. Lett. B 678, 246 (2009).

[21] A. Brandhuber, B. Spence, and G. Travaglini, From trees to loops and back, J. High Energy Phys. 01 (2006) 142.

[22] R. Britto and E. Mirabella, Single cut integration, J. High Energy Phys. 01 (2011) 135.

[23] D. Vaman and Y. P. Yao, QCD recursion relations from the largest time equation, J. High Energy Phys. 04 (2006) 030.

[24] M. Veltman, Unitarity and causality in a renormalizable field theory with unstable particles, Physica (Utrecht) 29, 186 (1963).

[25] D. Vaman and Y. P. Yao, Analytic acattering amplitudes for QCD, Mod. Phys. Lett. A 23, 847 (2008).

[26] R. P. Feynman, Quantum theory of gravitation, Acta Phys. Pol. 24, 697 (1963).

[27] R. P. Feynman, Closed loop and tree diagrams, in Selected Papers of Richard Feynman, edited by L. M. Brown (World Scientific, Singapore, 2000).

[28] S. Catani, T. Gleisberg, F. Krauss, G. Rodrigo, and J. C. Winter, From loops to trees by-passing Feynman's theorem, J. High Energy Phys. 09 (2008) 065.

[29] S. Caron-Huot, Loops and trees, J. High Energy Phys. 05 (2011) 080.
[30] I. Bierenbaum, S. Catani, P. Draggiotis, and G. Rodrigo, A tree-loop duality relation at two loops and beyond, J. High Energy Phys. 10 (2010) 073.

[31] C. Baadsgaard, N. E. J. Bjerrum-Bohr, J. L. Bourjaily, S. Caron-Huot, P. H. Damgaard, and B. Feng, New Representations of the Perturbative S-Matrix, Phys. Rev. Lett. 116, 061601 (2016).

[32] M. Maniatis, Scattering amplitudes abandoning virtual particles, arXiv:1511.03574.

[33] M. Maniatis, Application of the Feynman-tree theorem together with BCFW recursion relations, Int. J. Mod. Phys. A 33, 1850042 (2018).

[34] M. Maniatis and C. M. Reyes, Scattering amplitudes from a deconstruction of Feynman diagrams, arXiv:1605.04268.

[35] Z. Bern and D. A. Kosower, The computation of loop amplitudes in gauge theories, Nucl. Phys. B379, 451 (1992).

[36] S. D. Badger, E. W. N. Glover, V. V. Khoze, and P. Svrcek, Recursion relations for gauge theory amplitudes with massive particles, J. High Energy Phys. 07 (2005) 025 .

[37] N. Arkani-Hamed, T. C. Huang, and Y.t. Huang, Scattering amplitudes for all masses and spins, arXiv:1709.04891. 